\title{
LXXVI. Note on the theory of radiation
}

\section{C.G. Darwin}

To cite this article: C.G. Darwin (1922) LXXVI. Note on the theory of radiation, Philosophical Magazine Series 6, 43:256, 641-648, DOI: 10.1080/14786442208633923

To link to this article: http://dx.doi.org/10.1080/14786442208633923

册 Published online: 08 Apr 2009.

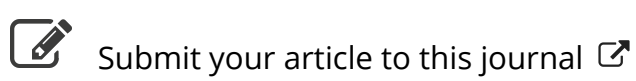

Џll Article views: 5

Q View related articles $₫$ 
LONDON, KDINBURGH, AND DUBLIN

\title{
PHILOSOPHICAL MAGAZINE
}

\author{
AND \\ JOURNAL OF SCIENCE.
}

[BIXTH SERIEB.]

APRIL 1922.

LXXVI. Note on the Theory of Radiation. By C. G. DarwIN, Fellow and Lecturer of Christ's College, Cambridge*

1. THE theory of optics and the thermodynamic theory 1 of radiation both deal with the electromagnetic changes of free space, and yet their methods of treatment are so widely different that hardly any point of contact is to be found in their fundamental definitions and procedure. In optics the primary description is in terms of the electric and magnetic forces, and an arbitrary field is specified by giving those forces at all times and places. On the other hand, in thermodynamics the analysis is entirely different, since it is in terms of radiant energy, passing through every point in every direction and sorted out according to its frequency. Now, though the spectroscope makes it fairly clear what is meant by "the energy in a certain range of frequencies," yet a rigorous definition is lacking, and even the conception of streams of radiation going simultaneously in all directions does not lend itself at once to the ordinary energy formulæ of electromagnetic theory. It appeared to me, therefore, that it might be useful to place on record the formal connexion between the optical and the thermodynamic descriptions of an arbitrary field of radiation; its outline must have been present in the minds of most writers on radiation, but $I$ have not seen it in any work on the subject.

* Communicated by the Author.

Phil. Mag. S. 6. Vol. 43. No. 256. April 1922. 
It should be said at once that it seems improbable that any practical use could be made of the relations, or even that an example of them could be given. For in an example it is only possible to specify the optical quantities by virtue of some regularity in their character, and this very regularity makes them unsuited for thermodynamic expression.

2. The only process by which the two types of quantity can be related consists in expressing as integrals the whole flow of energy across an area in terms of both, and then identifying the elements of the two integrands. In no other way can a rigorous definition be given for the thermodynamic quantities. The modus operandi consists in the repeated application of Fourier integrals. In the present note no pretence is made to mathematical rigour in the deductions, but there can, I think, be no doubt of their validity. The most important step in the process is based on a theorem, due originally to Stokes*, and used for the present purpose by Rayleigh $\dagger$. Stokes and Rayleigh both proved this theorem by introducing an exponential factor to help convergence. Here I have not used one, as it wonld probably be as hard to justify its introduction as to prove rigorously the result withont it. To save confusion with a more celebrated theorem, and because Stokes's work dealt with a rather different aspect, I shall call it Rayleigh's theorem. It will be convenient to exhibit it here first, so as to make its repeated use in the later work easier to follow.

Let

$$
\left.\begin{array}{l}
\phi_{\nu}=2 \int_{-\mathrm{T} / 2}^{\mathrm{T} / 2} \mathrm{Z}(t) \cos 2 \pi \nu t d t \\
\psi_{\nu}=2 \int_{-\mathrm{T} / 2}^{\mathrm{T} / 2} \mathrm{Z}(t) \sin 2 \pi \nu t d t,
\end{array}\right\}
$$

where $Z$ is a function of $t$, which is restricted in such a way that $\phi_{\nu}$ and $\psi_{\nu}$ are continuous, and vanish for large values of $v$ according to some appropriate rule of convergence (the sequel suggests that the necessary condition is $\left.\phi_{\nu}, \psi_{\nu}=0\left(\nu^{-\frac{1}{2}-\epsilon}\right), \epsilon>0\right)$, while they vanish for $\nu=0$. T is so large that for $\nu=0(1 / T), \phi_{\nu}$ and $\psi_{\nu}$ are negligible.

By the principle of the inversion of Fourier integrals, if

$$
\mathrm{Z}_{1}=\int_{0}^{\infty}\left(\phi_{\nu} \cos 2 \pi \nu t+\psi_{\nu} \sin 2 \pi \nu t\right) d \nu, .
$$

then $\mathrm{Z}_{1}=\mathrm{Z}$ in the interval $\pm \mathrm{T} / 2$ and vanishes outside it.

* Stokes, Edinb. Trans. xx. p. 317 (1852).

† Rayleigh, Phil. Mag. xxvii. p. 460 (188y). 
Let $\overline{Z^{2}}$ be the mean of the square of $\mathrm{Z}$ taken over the range $\pm \mathrm{T} / 2$. Then

$$
\begin{aligned}
\overline{Z^{2}} & =\frac{1}{\mathrm{~T}} \int_{-\mathrm{T} / 2}^{\mathrm{T} / 2} \mathrm{Z}_{1}^{2} d t \\
& =\frac{1}{\mathrm{~T}} \int_{-T / 2}^{\mathrm{T} / 2} d t \int_{0}^{\infty} d \nu \int_{0}^{\infty} \begin{array}{r}
d \nu^{\prime}\left(\phi_{\nu} \cos 2 \pi \nu t+\psi_{\nu} \sin 2 \pi \nu t\right) \\
\times\left(\phi_{\nu^{\prime}} \cos 2 \pi \nu^{\prime} t+\psi_{\nu^{\prime}} \sin 2 \pi \nu^{\prime} t\right) .
\end{array}
\end{aligned}
$$

With the restrictions on $\phi_{\nu}$ and $\psi_{\nu}$ it is permissible to invert the order of integration, and to lake that for $t$ first. Then

$$
\begin{aligned}
\overline{Z^{2}}=\frac{1}{2 \Psi_{1}} \int_{0}^{\infty} d \nu \int_{0}^{\infty} d \nu^{\prime}\{ & \left(\phi_{\nu} \phi_{\nu^{\prime}}+\psi_{\nu} \psi_{\nu^{\prime}}\right) \frac{\sin \pi\left(\nu^{\prime}-\nu\right) \mathrm{T}}{\pi\left(\nu^{\prime}-\nu\right)} \\
& \left.+\left(\phi_{\nu} \phi_{\nu^{\prime}}-\psi_{\nu} \psi_{\nu^{\prime}}\right) \frac{\sin \pi\left(\nu^{\prime}+\nu\right)^{\prime} \mathrm{I}^{\prime}}{\pi\left(\nu^{\prime}+\nu\right)}\right\} .
\end{aligned}
$$

Following the usual reasoning for Fourier integrals, with the conditions imposed on $\mathrm{T}$ the important part of this is the first term, and for it $\nu^{I}$ can be changed into $\nu$ in the first factor. Performing the integration for $v^{\prime}$, we then have

$$
Z^{\overline{2}}=\frac{1}{2 \overline{\mathrm{T}}} \int_{0}^{\infty}\left(\phi_{v}{ }^{2}+\psi_{\nu}{ }^{2}\right) d \nu, . . .
$$

which is the theorem.

There is one point in this result that deserves mention, and that is the presence of the factor $1 / \mathrm{T}$. For at first sight this suggests that if the time considered were long enough, the average would tend to vanish. This is not so, because the magnitades of $\phi_{\nu}$ and $\psi_{v}$ will vary with T by $(2 \cdot 1)$. Still if $\mathrm{Z}$ were quite arbitrary, $\overline{\mathrm{Z}^{2}}$ would naturally depend on the exact value of $T$. The utility of $(2 \cdot 3)$ depends on its application to rather more specialized typus of function. For use in radiation theory we must attribute to $\mathrm{Z}$ the property which the radiation, in fact, has-that $\bar{Z}^{2}$ has a value independent of the exact value of $\mathrm{T}$, and $\phi_{v}$ and $\psi_{\nu}$ will then be proportional to $\mathrm{T}^{1 / 2}$. But it does not seem possible to make $\mathrm{T}$ disappear from the expression $(2 \cdot 3)$.

3. The radiation field is specified in the optical manner by means of the six components of electric and magnetic force. To derive from these the thermodynamic description it is necessary first to express the optical quantities as a set of plane waves going in all direetions; then the Poynting vector must be calculated from these, and must be integrated $2 \mathrm{~T} 2$ 
so as to give the total flow of energy through a given aperture in a given time. By successive applications of Rayleigh's theorem the result can be brought to an integral of the same form as the corresponding thermodynamic expression, and the interrelation is then given by equating the two integrands.

Let the optical field be given by X, Y, Z; $\alpha, \beta, \gamma$, which are arbitrary functions of $x, y, z, t$, subject to their satisfying the electromagnetic equations and to suitable conditions as to continuity and convergence. We shall evaluate the total radiation crossing the $y z$ plane between $y= \pm p / 2$ and $z= \pm q / 2$ daring the time between $t= \pm \mathrm{T} / 2$. Here $p$ and $q$ are to be so large that they include many wave-lengths of the types of waves that occur, and the same must be true for $T$ and the periods. On the other hand, they must be sufficiently small to allow for the gross variations of radiation in the different parts of space. These limitations are like those which are imposed in the kinetic theory of gases. More precisely the functions $\mathrm{X}, \mathrm{Y}, \mathrm{Z} ; \alpha, \beta, \gamma$ are such that the averages to be constructed shall be independent of $p, q$, and $T$. It should be said that this independence will not be apparent formally, bat will occur in the same way as in $(2 \cdot 3)$.

4. We proceed to consider how the radiation is to be analysed into plane waves. A typical plane wave is described by the expression

where

$$
S=g_{1}^{s} \cos \tau+g_{2}^{8} \sin \tau,
$$

$$
\tau=2 \pi \nu\{t-(x \sin \theta \cos \phi+y \sin \theta \sin \phi+z \cos \theta) / c\},
$$

while $\mathrm{S}$ and $g^{s}$ represent in turn each of the components of electric and magnetic force according to the rule

$g^{\mathrm{x}}=\mathrm{U} \cos \theta \cos \phi-\mathrm{V} \sin \phi \quad g^{\alpha}=-\mathrm{U} \sin \phi-\mathrm{V} \cos \theta \cos \phi$ ? $\left.\begin{array}{ll}g^{\mathrm{Y}}=\mathrm{U} \cos \theta \sin \phi+\mathrm{V} \cos \phi & g^{\beta}=\mathrm{U} \cos \phi-\mathrm{V} \cos \theta \sin \phi \\ g^{\mathrm{Z}}=-\mathrm{U} \sin \theta & g^{\gamma}=\mathrm{V} \sin \theta\end{array}\right\}$

Here $U$ corresponds to the wave polarized with electric force in a plane through the $z$ axis and $\mathrm{V}$ to that in the perpendicular direction.

The arbitrary field can be represented by taking four quantities $\mathrm{U}_{1}, \mathrm{~V}_{1}, \mathrm{U}_{2}, \mathrm{~V}_{2}$ as functions of $v, \theta, \phi$ and expressing any of the forces as

$$
\mathrm{S}=\int_{0}^{\infty} d \nu \int_{0}^{\pi} \sin \theta d \theta \int_{0}^{2 \pi} d \phi\left(g_{1}^{s} \cos \tau+g_{2}{ }^{8} \sin \tau\right)
$$


As we are to consider the flow of energy through the aperture in the $y z$ plane, we put $x=0$ in this expression, thus changing $\tau$ into

$$
\tau_{0}=2 \pi \nu\{t-(y \sin \theta \sin \phi+z \cos \theta) / r\} .
$$

But now a difficulty arises in determining the $g$ 's, for no one of the forces by itself can determine whether the wave is going forwards or backwards. This difficulty is reflected in the mathematics, for in the $\phi$ integration $\tau_{0}$ assumes all its values twice over, which prevents the usual inversion. To obviate this, we separate out and add together the $g$ 's corresponding to $\phi$ and $\pi-\phi$ and take the $\phi$ integration between $\pm \pi / 2$. Thus, if

$$
\mathrm{G}^{s}=g^{s}(\nu, \theta, \phi)+g^{s}(\nu, \theta, \pi-\phi),
$$

we take as the typical formula

$$
S_{1}=\int_{0}^{\infty} d \nu \int_{0}^{\pi} \sin \theta d \theta \int_{-\pi 2}^{\pi_{2}^{2}} d \phi\left(G_{1}^{s} \cos \tau_{0}+G_{\pi_{2}}^{s} \sin \tau_{0}\right) .
$$

These considerations show what formule are aimed at. To obtain them put

$\mathrm{G}_{1}{ }^{s}=2 \frac{v^{2}}{\nu^{2}} \sin \theta \cos \phi \int_{-p, 2}^{p, 2} d y \int_{-q / 2}^{q, 2} d z \int_{-\mathrm{T}_{/ 2}}^{T_{i} 2} d t \mathrm{~S}(0, y, z, t) \cos \tau_{0}$.

and a similar expression in $\mathrm{G}_{2}{ }^{\mathrm{s}}$ and $\sin \tau_{0}$. Then, by three successive inversions for $\phi, \theta$, and $\nu$ in turn, it may be shown that $\mathrm{S}_{1}=\mathrm{S}$ between the limits $\pm p / 2, \pm q / 2, \pm \mathrm{T} / 2$ and vanishes outside them. Knowing the $G_{1}$ 's we are thus led to six equations of the type of $(4 \cdot 2)$, of which the first is, for instance,

$$
\begin{aligned}
\mathrm{G}_{1}{ }^{\mathrm{X}}=\left[\mathrm{U}_{1}(\phi)\right. & \left.-\mathrm{U}_{1}(\pi-\phi)\right] \cos \theta \cos \phi \\
& -\left[\mathrm{V}_{1}(\phi)+\mathrm{V}_{2}(\pi-\phi)\right] \sin \phi .
\end{aligned}
$$

and there are six similar equations in $\mathrm{G}_{2}, \mathrm{U}_{2}, \mathrm{~V}_{2}$. The U's and V's can be determined from these, and give

$$
\left.\begin{array}{l}
\mathrm{U}(\phi)=\left\{-\mathrm{G}^{\mathrm{Z}} \cos \phi+\mathrm{G}^{\beta} \sin \theta+\mathrm{G}^{\mathrm{y}} \cos \theta \sin \phi\right\} / 2 \sin \theta \cos \phi \\
\mathrm{V}(\phi)=\left\{\mathrm{G}^{\mathrm{y}} \cos \phi+\mathrm{G}^{\mathrm{Y}} \sin \theta+\mathrm{G}^{\mathrm{Z}} \cos \theta \sin \phi\right\} / 2 \sin \theta \cos \phi
\end{array}\right\} .
$$

There are similar equations for $U(\pi-\phi)$ and $V(\pi-\phi)$ and two redundant equations of the form

$$
\left.\begin{array}{rl}
\mathrm{G}^{\mathrm{x}} & =\mathrm{G}^{\beta} \cos \theta-\mathrm{G}^{\gamma} \sin \theta \sin \phi \\
-\mathrm{G}^{\alpha} & =\mathrm{G}^{\mathrm{Y}} \cos \theta-\mathrm{G}^{\mathrm{z}} \sin \theta \sin \phi
\end{array}\right\}, \quad .
$$

which must be satisfied identically. That they are, may be 
seen from the fact that, expressed in terms of the forces $X$ etc. by $(4 \cdot 4)$, they are simply those two of the electromagnetic equations which do not involve the operator $\partial / \partial x-$ that is, which are valid on the $y z$ plane.

5. We next find the flow of energy through the aperture, and apply Rayleigh's theorem to it. The flow in the positive direction of $x$ per unit area per unit time is the component of the Poynting vector $\left(\mathrm{Y}_{\gamma}-\mathrm{Z} \beta\right) c / 4 \pi$, and so the total flow through the aperture in time $\mathrm{T}$ is $\mathrm{F}$, where

$$
\begin{aligned}
\mathrm{F}= & \frac{c}{4 \pi} \int_{-p / 2}^{p / 2} d y \int_{-q^{\prime 2}}^{q / 2} d z \int_{-\mathrm{T} / 2}^{\mathrm{T} / 2} d t\left(\mathrm{Y}_{1} \gamma_{1}-\mathrm{Z}_{1} \beta_{1}\right) \\
= & \frac{r}{4 \pi} \int_{-p / 2}^{p / 2} d y \int_{-q / 2}^{q / 2} d z \int_{-\mathrm{T} / 2}^{\mathrm{T} / 2} d t \int_{0}^{\infty} d \nu \int_{0}^{\pi} \sin \theta d \theta \int_{-\pi / 2}^{\pi / 2} d \phi \\
& \left.\int_{0}^{\infty} d \nu^{\prime}\right|_{0} ^{\pi} \sin \theta^{\prime} d \theta^{\prime} \int_{-\pi / 2}^{\pi / 2} d \phi^{\prime} \\
& {\left[\left\{\mathrm{G}_{1}{ }^{\mathrm{Y}} \cos \tau_{0}+\mathrm{G}_{2}{ }^{\mathrm{I}} \sin \tau_{0}\right\}\left\{\mathrm{G}_{1}{ }^{\gamma^{\prime}} \cos \tau_{0}{ }^{\prime}+\mathrm{G}_{2}{ }^{\prime \prime} \sin \tau_{0}{ }^{\prime}\right\}\right.} \\
& \left.-\left\{\mathrm{G}_{1}{ }^{\mathrm{Z}} \cos \tau_{0}+\mathrm{G}_{2}{ }^{\mathrm{Z}} \sin \tau_{0}\right\}\left\{\mathrm{G}_{1}{ }^{\beta^{\prime}} \cos \tau_{0}{ }^{\prime}+\mathrm{G}_{2}{ }^{\beta^{\prime}} \sin \tau_{0}{ }^{\prime}\right\}\right]
\end{aligned}
$$

In this $G_{1}{ }^{\beta}$ is the same function as $G_{1}{ }^{\beta}$, but with arguments $v^{\prime}, \theta^{\prime}, \phi^{\prime}$, and $\tau_{0}^{\prime}$ is the same as $\tau_{0}$, but with arguments $\nu^{\prime}, \theta^{\prime}, \phi^{\prime}, y, z, t$.

To avoid writing out many terms which will afterwards vanish in the integrations, observe that in this expression only those arising out of $\frac{1}{2} \cos \left(\tau_{0}-\tau_{0}{ }^{\prime}\right)$ will contribute anything, and that those consist of the sum of identical terms in $G_{1}$ 's and $G_{2}$ 's. We shall therefore only consider the former. Reverse the order of the integrations and perform those for $y, z, t$; then

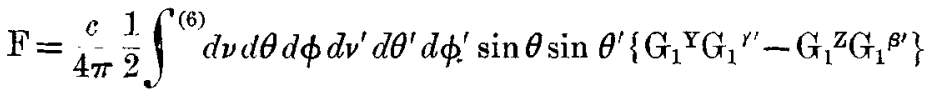

$$
\begin{aligned}
& \frac{\sin \pi\left(\nu^{\prime}-\nu\right) \mathrm{T}}{\pi\left(\nu^{\prime}-\nu\right)} \frac{\sin \pi\left(\nu^{\prime} \sin \theta^{\prime} \sin \phi^{\prime}-\nu \sin \theta \sin -\jmath\right) p / r^{\prime}}{\pi\left(\nu^{\prime} \sin \theta^{\prime} \sin \phi^{\prime}-\nu \sin \theta \sin \phi\right) / c} \\
& \frac{\sin \pi\left(v^{\prime} \cos \theta^{\prime}-\nu \cos \theta\right) g / c}{\pi\left(\nu^{\prime} \cos \theta^{\prime}-\nu \cos \theta\right) / c} \text { : }
\end{aligned}
$$

Now integrate for $\nu^{\prime}$. This removes the first factor of the second line and changes $\nu^{\prime}$ into $\nu$ in the rest. Next, the $\theta^{\prime}$ integration removes the factor in the third line, giving a multiplier $c / v \sin \theta$ and changing $\theta^{\prime}$ into $\theta$ in the rest. Then 
the $\phi^{\prime}$ integration removes the middle factor, giving a multiplier $c / \nu \sin \theta \cos \phi$ and changing $\phi^{\prime}$ into $\phi$. Thus

$\mathrm{F}=\frac{c}{8 \pi} \int_{0}^{\infty} d \nu \int_{0}^{\pi} d \theta \int_{-\pi / 2}^{\pi / 2} d \phi\left\{\mathrm{G}_{1}{ }^{\mathrm{Y}} \mathrm{G}_{1}{ }^{\gamma}-\mathrm{G}_{1} \mathrm{Z}^{\mathrm{Z}} \mathrm{G}_{1}{ }^{\beta}\right\} c^{2} / \nu^{2} \cos \phi .(5 \cdot 3)$

Now substitute for the $G_{1}^{\prime} \mathrm{g}$ in terms of $U_{1}$ and $V_{1}$. By $(4 \cdot 2)$ and $(4 \cdot 5)$

$$
\begin{aligned}
\mathrm{G}_{1}{ }^{\mathrm{Y}} \mathrm{G}_{1}{ }^{\gamma}-\mathrm{G}_{1}{ }^{Z \mathrm{G}_{1}{ }^{\beta}} & =\sin \theta \cos \phi\left[\mathrm{U}_{1}{ }^{2}(\phi)+\mathrm{V}_{1}{ }^{2}(\phi)\right. \\
& \left.-\mathrm{U}_{1}{ }^{2}(\pi-\phi)-\mathrm{V}_{1}{ }^{2}(\pi-\phi)\right] .
\end{aligned}
$$

So if we denote $\mathrm{U}_{1}{ }^{2}+\mathrm{U}_{2}{ }^{2}$ by $\mathrm{U}^{2}$, we have finally

$$
\begin{array}{r}
\mathrm{F}=\frac{c^{3}}{8 \pi} \int_{0}^{\infty} \frac{d \nu}{\nu^{2}} \int_{0}^{\pi} \sin \theta d \theta \int_{-\pi / 2}^{\pi / 2} d \phi\left[\mathrm{U}^{2}(\phi)+\mathrm{V}^{2}(\phi)\right. \\
\left.-\mathrm{U}^{2}(\pi-\phi)-\mathrm{V}^{2}(\pi-\phi)\right] .
\end{array}
$$

6 . For the thermodynamic treatment of radiation * we take an aperture $d \sigma$, and from it, at angle $\epsilon$ to the normal, draw a cone of small solid angle $d \omega$. The energy passing through the aperture into this cone in time $d t$ is supposed separated into its two components of polarization and analysed into its frequencies, so that the specification of the field is

$$
\left(\mathrm{K}_{\nu}+\mathrm{K}_{\nu}^{\prime}\right) d \nu d \sigma \cos \epsilon d \omega d t . \quad \text {. . . }
$$

Here we are to take

$$
d \sigma=p q, \quad \cos \epsilon=\sin \theta \cos \phi, \quad d \omega=\sin \theta d \theta d \phi, \quad d t=\mathrm{T} .
$$

Let $K_{v}$ refer to the component polarized with electric force in a plane through the $z$ axis. Then the whole excess of energy going in the positive direction is

$$
\begin{array}{r}
\mathrm{F}=p q^{\mathrm{T}} \int_{0}^{\infty} d \nu \int_{0}^{\pi} \sin \theta d \theta \int_{-\pi / 2}^{\pi / 2} d \phi\left[\mathrm{K}_{\nu}(\phi)+\mathrm{K}_{\nu}^{\prime}(\phi)\right. \\
\left.-\mathrm{K}_{\nu}(\pi-\phi)-\mathrm{K}_{\nu}^{\prime}(\pi-\phi)\right] \sin \theta \cos \phi .
\end{array}
$$

If we equate the terms of this integrand to those of $(5 \cdot 5)$, we have

$$
\left.\begin{array}{l}
\mathrm{K}_{\nu}=c^{3} \mathrm{U}^{2} / 8 \pi \nu^{2} \cdot \sin \theta \cos \phi \cdot p q \mathrm{~T} \\
\mathrm{~K}_{\nu}{ }^{\prime}=c^{3} \mathrm{~V}^{2} / 8 \pi \nu^{2} \cdot \sin \theta \cos \phi \cdot p q \mathrm{~T}
\end{array}\right\},
$$

and these constitute the formal definitions of $K_{v}$ and $K_{v}$ '.

Just as in $\S 2$ we saw that $\overline{Z^{2}}$ involved $T$ explicitly, though

* See Planck, Vorlesungen über die Theorie der Wärmestrahlung (Barth). Many of the relations, which he proves with some difficulty by Fourier series, come out much easier with Fourier integrals. 
really it would be independent, so here $K_{\nu}$ appears to involve $p, q, \mathrm{~T}$. It also involves the factor $\sin \theta \cos \phi$ and at first sight it seems that this should have disappeared in the course of the calculations, since it arises from the $\cos \epsilon$ in $\left(6^{\circ} 1\right)$ and simply means that the cone of rays may be cut by a plane in any direction to give the aperture. But, in effect, this corresponds to taking a new aperture-that is, new values for $p$ and $q$,-and as it is not possible to verify that such a change is immaterial even when the plane of the aperture is unchanged, a fortiori, it will not be possible when the plane is changed as well. We can only say that if a real radiation field were specified in the optical manner, and if the calculations of $\$ \S 4,5$, and 6 were applied to it, we should find that the resulting $K_{\nu}$ was independent of the time and of both the area and direction of the aperture.

If the values of $U$ and $V$ are written in from $(4 \cdot 6)$ and $(4 \cdot 4)$, we have in full

$$
\begin{aligned}
\mathrm{K}_{\mathrm{u}}= & \frac{1}{p q^{\prime} \mathrm{T} \sin \theta \cos \phi} \nu^{2} \int_{-p / 2}^{p / 2} d y \int_{-q / 2}^{q / 2} d z \int_{-\mathrm{T} / 2}^{\mathrm{T} / 2} d t \int_{-p / 2}^{p / 2} d y^{\prime} \int_{-q / 2}^{q / 2} d z^{\prime} \\
& \int_{-\mathrm{T} / 2}^{\mathrm{T} / 2} d t^{\prime} \cos 2 \pi \nu\left\{\left(t-t^{\prime}\right)-\left[\left(y-y^{\prime}\right) \sin \theta \sin \phi+\left(z-z^{\prime}\right) \cos \theta\right] / e\right\} \\
& \times(-\mathrm{Z} \cos \phi+\beta \sin \theta+\gamma \cos \theta \sin \phi) \\
& \times\left(-\mathrm{Z}^{\prime} \cos \phi+\beta^{\prime} \sin \theta+\gamma^{\prime} \cos \theta \sin \phi\right), \quad . \quad\left(t^{\circ} \cdot 4\right)
\end{aligned}
$$

where $Z^{\prime}$, etc. are the same as $Z$, etc., taken with arguments $y^{\prime}, z^{\prime}, t^{\prime}$. For $\mathbf{K}_{\nu}^{\prime}$ the brackets are

$$
(\gamma \cos \phi+Y \sin \theta+Z \cos \theta \sin \phi), \text { etc. }
$$

and otherwise the expression is the same. The relation $(6 \cdot 4)$ can be put into other forms by virtue of $(4 \cdot 7)$. A particularly simple case is that of the radiation going normally from the aperture, which is found by putting $\theta=\pi / 2, \phi=0$. $\mathrm{K}_{\nu}^{x, z}$ signifies the $\mathrm{K}_{\nu}$ of the ray going along the $x$ axis and polarized with electric force in the $x z$ plane.

$$
\begin{array}{r}
\mathrm{K}_{\nu} x_{*}=\frac{1}{p q \mathrm{~T}} \frac{\nu^{2}}{8 \pi c} \int_{-p / 2}^{p / 2} d y \int_{-q^{\prime 2}}^{q / 2} d z \int_{-\mathrm{T} / 2}^{\mathrm{T} / 2} d t \int_{-p / 2}^{p / 2} d y^{\prime} \int_{-q / 2}^{q / 2} d z^{\prime} \\
\int_{-\mathrm{T} / 2}^{\mathrm{T} / 2} d t^{\prime} \cos 2 \pi \nu\left(t-t^{\prime}\right)(\beta-\mathrm{Z})\left(\beta^{\prime}-\mathrm{Z}^{\prime}\right) .
\end{array}
$$

For $K_{\nu}^{x, y}$ the last two factors must be replaced by $(\gamma+Y)$ $\left(\gamma^{\prime}+\mathrm{Y}^{\prime}\right)$.

This complates the problem of formally expressing the thermodynamic specification of the field in terms of the optical. 